\title{
Análise da associação entre o quadro clínico referido pelas pacientes portadoras de endometriose e o local de acometimento da doença
}

\author{
Analysis of the association between \\ symptoms referred by patients with \\ endometriosis and the site \\ of the disease
}
Bruna Thomazelli Berbel'1, Sérgio Podgaec², Maurício Simões Abrão ${ }^{3}$

\begin{abstract}
Berbel BT, Podgaec S, Abrão MS. Análise da associação entre o quadro clínico referido pelas pacientes portadoras de endometriose e o local de acometimento da doença. Rev Med (São Paulo). 2008 jul.-set.;87(3):195-200.
\end{abstract}

\begin{abstract}
RESUMO: A endometriose é uma doença caracterizada pelo implante ectópico de estroma e/ou epitélio glandular endometrial em localização extra-uterina que acomete $15 \%$ das mulheres em idade reprodutiva, podendo se manifestar em focos superficiais peritoneais, no ovário ou na forma infiltrativa profunda. A paciente pode apresentar quadro clínico diverso com queixa de dispareunia de profundidade, dismenorréia, dor pélvica acíclica, infertilidade, alterações intestinais e urinárias cíclicas. A suspeita diagnóstica é feita através de quadro clínico sugestivo associado com exames de imagem, porém a confirmação depende de procedimento cirúrgico, como a vídeo-laparoscopia que permite visualização da pelve e obtenção de amostra tecidual para posterior análise histológica.

Em algumas pesquisas acerca do atraso no diagnóstico da endometriose desde o aparecimento de sintomas, concluiu-se que uma das principais causas desse atraso é o pouco conhecimento relativo à doença entre pacientes e médicos, além da dificuldade de acesso à cirurgia laparoscópica em serviços públicos de saúde. Algumas pesquisas foram feitas para tentar relacionar os tipos de doença, sintomas e locais acometidos e acharam associações mais evidentes entre dispareunia de profundidade e doença em fundo de saco posterior, além de maior intensidade de dismenorréia e dor pélvica acíclica em estádios mais avançadas da doença. Nosso estudo faz uma revisão da literatura no sentido de encontrar relações consistentes entre as características da doença e os sintomas, buscando vieses que prejudicaram outras pesquisas, como de seleção de pacientes e informação, e permitindo à prática clínica o diagnóstico mais precoce das pacientes.
\end{abstract}

DESCRITORES: Infertilidade. Dor pélvica. Endometriose.

1. Acadêmica do curso de Medicina da Faculdade de Medicina da Universidade de São Paulo.

2. Mestre em Ginecologia pela Faculdade de Medicina da Universidade de São Paulo, Médico do Setor de Endometriose do Departamento de Ginecologia do Hospital das Clínicas da Faculdade de Medicina da Universidade de São Paulo.

3. Professor Livre-Docente de Ginecologia pela Faculdade de Medicina da Universidade de São Paulo, Médico Responsável pelo Setor de Endometriose do Departamento de Ginecologia do Hospital das Clínicas da Faculdade de Medicina da Universidade de São Paulo.

Endereço para correspondência: Avenida Doutor Enéas de Carvalho Aguiar 255, Cerqueira César. São Paulo, SP. CEP: 05403-900. 


\section{INTRODUÇÃO}

A endometriose é uma doença caracterizada pelo implante ectópico de estroma e/ou epitélio glandular endometrial em localização extra-uterina podendo comprometer diversos locais, como ligamentos úterosacros, ovários e tratos urinário e estima-se que 10 a $15 \%$ das mulheres em idade reprodutiva e $3 \%$ na pós-menopausa apresentem essa doença ${ }^{13}$.

Estudo realizado no Setor de Endometriose do Hospital das Clínicas da Faculdade de Medicina da Universidade de São Paulo demonstrou que mais de $50 \%$ das pacientes atendidas tinham escolaridade acima do segundo grau completo evidenciando que provavelmente, um grande número de mulheres afetadas e de nível sócio-econômico mais baixo não procuram atendimento e não são inseridas nos estudos ${ }^{1}$. Mulheres com infertilidade também podem ser causa de erro nos estudos epidemiológicos, pois somente as que têm desejo de engravidar e procuram tratamento serão diagnosticadas. Por isso, alguns autores defendem a idéia de que pacientes assintomáticas, com endometriose mínima, não devem ser envolvidas em estudos sobre doença sintomática ou severa ${ }^{22}$. Todos esses fatores, entre outros podem influenciar no cálculo estatístico de prevalência e incidência.

\section{ETIOPATOGENIA}

Algumas teorias foram propostas para explicar o desenvolvimento da doença. As duas correntes principais sobre a etiopatogenia da endometriose são: a da metaplasia celômica ${ }^{15}$ e da menstruação retrógrada ${ }^{19}$. A primeira afirma que o mesotélio poderia se transformar em tecido endometrial, o que explicaria o implante ectópico em áreas extrauterinas. Já a teoria da menstruação retrógrada postula que existe refluxo menstrual pelas trompas até a cavidade abdominal, e lá o endométrio poderia se instalar causando a doença. Ambas as teorias têm sido criticadas e reformuladas por alguns estudos agregando a elas novos conhecimentos.

Estuda-se bastante o papel do sistema imune nessa doença já que, teoricamente, as células do endométrio que se implantam fora do seu local habitual de desenvolvimento deveriam ser fagocitadas ou destruídas por células de defesa do organismo. Em mulheres afetadas, a varredura dessas células não deve ocorrer de forma eficiente, o que permite o desenvolvimento da endometriose ${ }^{14}$. Alguns estudos postulam que, nos estádios iniciais, para que o endométrio consiga se implantar, a resposta celular do sistema imunológico ou resposta Th1 deve ser inibida para que a citotoxicidade dos linfócitos não a impeça de se instalar. Já em estádios mais avançados da doença, com quadros bastante agressivos e infiltrativos, a resposta celular se tornaria mais evidente a fim de proteger o organismo contra uma doença que já poderia estar prejudicando diversos órgãos ${ }^{18}$. Foi demonstrado que existe diminuição da citotoxicidade de linfócitos contra o endométrio autólogo em pacientes doentes e, além disso, a concentração de algumas citocinas no líquido peritoneal dessas mulheres também se mostra mais elevada ${ }^{21}$ o que indica que a atuação do sistema imune deve ter papel importante no desenvolvimento da doença ${ }^{5}$.

\section{Tipos de endometriose}

Em 1997, foi lançado um conceito que dividiu a endometriose em três tipos de doença de acordo com os sítios acometidos e os tipos de lesões que ocorriam de acordo com o estágio da doença ${ }^{17}$. Assim, dividiram-na em ovariana, peritoneal e infiltrativa profunda. A peritoneal é a mais superficial, a ovariana inclui os cistos denominados endometriomas e os implantes superficiais do ovário e a infiltrativa profunda é a que apresenta profundidade maior que $5 \mathrm{~mm}$ e que acomete as regiões retro-cervical, paracervical, septo reto-vaginal, reto-sigmóide, ureteres e bexiga. As lesões da endometriose em estádios iniciais da doença têm coloração vermelha como o endométrio, mas após algum tempo, essas células estimulam uma resposta inflamatória e adquirem coloração bem escura. A fibrose que ocorre após a inflamação torna a lesão clara e o tecido perde a função. Descobriu-se também, que o mesotélio do ovário pode invaginar dentro do córtex formando os endometriomas, cistos ovarianos com conteúdo achocolatado típico da doença.

Outra classificação proposta em 2000 dividiu a endometriose em dois tipos: a que se localiza nos ovários, trompas e peritônio pélvico, chamada endometriose, com lesões mais superficiais e quadro clínico mais ameno, e a de acometimento central na região retro-uterina, uterina ou recesso vesicovaginal chamada adenomiose ${ }^{16}$. Nessa divisão, a classificação não é feita pela profundidade da doença, mas sim pela expressão da atividade do endométrio, ou seja, se ocorre sangramento dependente de hormônios sexuais esteróides ou metaplasia de músculo liso. $\mathrm{Na}$ adenomiose existiria uma resposta fraca a progesterona e ocorreria a diferenciação de tecido conjuntivo e muscular liso, assim como 
a camada basal do endométrio no útero responde aos hormônios esteróides (ocorre metaplasia de miofibroblastos em estroma endometrial e viceversa). Já na endometriose existiria sangramento dependente de hormônio esteróide até o momento em que o sítio sofresse fibrose. Com isso, eles concluíram que a endometriose e a adenomiose tem respostas diferentes a esses hormônios, o que poderia ser mais um alvo no tratamento da doença.

Os locais mais freqüentemente acometidos são os ovários e ligamentos sacro-úteros ${ }^{7}$.

\section{QUADRO CLÍNICO}

Os principais sintomas da endometriose são: dor pélvica, que inclui dispareunia profunda, dismenorréia e dor acíclica, infertilidade e alterações intestinais e urinárias ciclícas. Além destes, em algumas mulheres, podem ocorrer outros sintomas e doença como cistites intersticiais, cálculos renais de repetição, vulvodinia, síndrome temporomandibular e fibromialgia ${ }^{13}$.

Uma das causas das dores poderia ser a proximidade dos implantes com nervos. Uma pesquisa mostrou que as porcentagens de mulheres com implantes profundos em áreas bastante inervadas sentiam mais dores que as mulheres com outros tipos de implantes de endométrio ${ }^{3}$. Também foi notado que implantes mais velhos têm maior chance de serem infiltrados por nervos, o que geraria mais dor. Foi visto em experiências utilizando ratos que os implantes ectópicos podem desenvolver um suprimento nervoso simpático e sensorial, apresentando conexão direta com a via de nervos esplâncnicos e nervo vago. A via de entrada com a medula espinhal se localiza no mesmo segmento de chegada com os nervos dos ureteres, e superior ao segmento de chegada dos nervos provenientes do canal vaginal e bexiga. Com isso, existe a possibilidade de que as hiperalgesias vaginais e dores nos ureteres estejam relacionadas à mecanismos neurais de dor 6 .

O diagnóstico da endometriose envolve o quadro clínico, utilização de marcadores e laparoscopia. Os marcadores são substâncias que tem sua concentração aumentada ou diminuída em determinadas épocas do ciclo menstrual e que servem como parâmetro para a avaliação do estágio da doença. Um dos mais usados é o CA-125, um marcador que tem concentrações elevadas quando a endometriose se encontra em estágios mais avançados². A laparoscopia é um procedimento cirúrgico que permite a visualização de lesões suspeitas e obtenção de amostras teciduais que podem confirmar o diagnóstico histológico da endometriose. O que indica a necessidade da realização deste ato cirúrgico é sem dúvida a anamnese da paciente para conseguir se retirar todas as informações possíveis sobre seu quadro, o exame físico e exames de imagem, quando apropriados.

Para tentar descobrir os motivos do atraso no diagnóstico da endometriose, foi feito um estudo no Brasil com pacientes que sofriam desta doença com confirmação cirúrgica ${ }^{4}$. Elas responderam a um questionário que continha informações sobre dados sociodemográficos, antecedentes ginecológicos e obstétricos, tipos de sintomas e há quanto tempo apareceram. Os resultados mostraram que e a idade de início dos sintomas é inversamente proporcional ao atraso. Em mulheres com menos de 19 anos o tempo entre o início dos sintomas e diagnóstico cirúrgico foi de 12,1 anos e em mulheres com mais de 30 anos o tempo decorrido foi de 3,3 anos. Há vários motivos para que isso ocorra. As pessoas pensam que no período da adolescência é normal que o ciclo não seja regular e que a presença de dores na época da menstruação é natural. Além disso, o exame físico em pacientes mais jovens é difícil principalmente se forem inativas sexualmente. $O$ método diagnóstico mais eficiente, a laparoscopia, também é uma das causas da demora no diagnóstico. Por ter alto custo, em alguns países em desenvolvimento a lista de espera para esse procedimento nos hospitais é longa, o que prolonga o tempo com sintomatologia exuberante das pacientes.

Em uma pesquisa sobre esse mesmo assunto analisou o tempo de decorrido entre o início dos sintomas e o diagnóstico de doença infiltrativa profunda ${ }^{10}$. Foi visto que pacientes com doença profunda e lesões no reto, bexiga e ureteres, apresentam um quadro clínico mais pronunciado e o tempo decorrido entre o aparecimento de sintomas e o diagnóstico era 5.21 (+/-5.6) anos. Já em pacientes onde a endometriose não era infiltrativa profunda, o tempo era de 3.9 $(+/-4.7)$ anos $(p<0,05)$. Portanto, percebe-se que existe maior dificuldade em diagnosticar doença profunda, infelizmente, o tipo de endometriose onde os sintomas são mais severos.

Em um trabalho ${ }^{9}$ foi analisada a endometriose profunda infiltrativa. As pacientes foram separadas em quatro grupos de acordo com o local de acometimento da doença: ligamento útero-sacral, vagina, bexiga e intestino. Elas responderam a um questionário de avaliação da dor pélvica antes e depois de se submeterem a cirurgia para a retirada dos focos. 
Percebeu-se que a retirada das lesões profundas pela cirurgia diminuiu a intensidade dos sintomas e melhorou a qualidade de vida dessas mulheres. Os resultados do trabalho mostram que tratamento no pré-operatório não traz grandes benefícios após a cirurgia, porém ainda se realiza o tratamento antes da cirurgia algumas vezes para diminuir o tamanho das lesões e facilitar a excisão ou torná-las inativas e menos vascularizadas, diminuindo a perda de sangue durante a intervenção.

Um estudo recente fez uma análise da associação entre as características das lesões, estágio da doença e severidade da dor em um grupo de 1054 pacientes $^{8}$. Cada paciente teve de responder a dois questionários que avaliavam a severidade $e$ as características da dor pélvica. A associação mais evidente encontrada foi entre as lesões de fundo de saco e a presença de dispareunia. Também se percebeu que a intensidade dessas dores decai com a idade, talvez porque a produção de estrogênio diminua assim como a atividade sexual. Foi visto que a intensidade da dor não é determinada pelo tipo ou extensão da lesão, mas sim pela sua relação com fibras nervosas, por isso, a dor referida pela paciente não deve servir como parâmetro para avaliar o estágio da doença. Uma análise parecida foi realizada com 160 mulheres com diagnóstico de endometriose por laparoscopia ou laparotomia que não tinham outras doenças pélvicas ${ }^{11}$. Elas também responderam a dois questionários sobre dor. Um multidimensional, que enfatiza o impacto social dos sintomas na vida das pacientes, e outro que continha uma escala analógica de dor, um pouco mais subjetivo. São usados dois tipos de questionário para garantir consistência ao trabalho, ou seja, evitar valores discrepantes nos resultados. O trabalho concluiu que não deve haver relação entre o estágio da doença, sítio de acometimento e severidade da doença, no entanto os próprios pesquisadores apontam que o número de pacientes utilizado é reduzido e, portanto, isso pode ter afetado os resultados já que são muitas variáveis a serem analisadas. Outro entrave que pode ter alterado resultados é que a endometriose ativa pode estar presente em peritônio aparentemente intacto para a laparoscopia. Sendo assim, mesmo sem variações perceptíveis da anatomia, podem existir sítios de produção de prostaglandinas que estão relacionadas aos sintomas pélvicos.

Para reavaliar os resultados obtidos neste estudo foi feito um novo somente com pacientes que sofriam de infertilidade, sendo que 124 tinham endometriose e 67 eram do grupo controle ${ }^{12}$. Foram excluídas pacientes com outras doenças pélvicas e cirurgias prévias. $O$ método com os questionários foi o mesmo do anterior e os resultados obtidos foram que a dismenorréia tem prevalência igual em todos os estágios, mas é mais severa em estágios mais avançados da doença; a dispareunia profunda é mais freqüente em mulheres com endometriose; a dor pélvica é mais freqüente em estágios mais avançados. Uma das limitações do estudo desta vez foi que as pacientes usadas não representam a população já que todas eram inférteis, porém, assegurou que todas pudessem ser comparadas pela idade, estado psicológico e história reprodutiva. Um estudo ideal deveria ser feito com mulheres da população em geral, mas isso não é possível, pois o método que garante o diagnóstico é invasivo. Questões financeiras e, principalmente, éticas estariam em jogo.

Em uma pesquisa feita recentemente, analisou-se a dor pélvica crônica e sua relação com a endometriose que é sua segunda maior causa $^{20}$. As 181 pacientes com dor pélvica crônica e endometriose se submeteram a laparoscopia, depois a um tratamento de seis meses com análogo de GnRH e novamente à laparoscopia. Após a segunda cirurgia um grupo foi tratado com anti-concepcional e o outro grupo foi usado como controle. Elas responderam a questionários sobre a dor antes da primeira e da segunda cirurgia. Os resultados obtidos foram de que a doença ovariana é a que está menos relacionada com a dor pélvica, porém, se o endometrioma for diagnosticado precocemente as chances de fertilidade aumentam bastante. A doença profunda é a mais relacionada com a dispareunia profunda, e como as massas retrocervicais respondem pouco a medicação por ter pouca quantidade de tecido estromal e glandular, o melhor tratamento nesses casos é cirúrgico. A principal conclusão do trabalho é de que os três tipos de doença (peritoneal, ovariana e infiltrativa profunda), têm causas diferentes e por isso, os tratamentos devem ser individualizados para cada tipo de caso.

Apesar de estes trabalhos terem sido feitos acerca da dismenorréia, dispareunia, dor pélvica e sua relação com diferentes tipos de endometriose não há nenhum estudo que aponte a prevalência de cada sintoma com o tipo de doença e com sua gravidade. Ao se verificar se há relação entre os sintomas referidos pelas pacientes e cada local de acometimento da endometriose, a suspeita diagnóstica deva se tornar mais ágil e, as pacientes poderão começar seus tratamentos para amenizar as dores e controlar o desenvolvimento dos implantes mais rapidamente. 
Berbel BT, Podgaec S, Abrão MS. Analysis of the association between symptoms referred by patients with endometriosis and the site of the disease. Rev Med (São Paulo). 2008 jul.-set.;87(3):195-200.

\begin{abstract}
Endometriosis is a disease characterized by ectopic implantation of stroma and/or endometrial glands in extra-uterine location that affects $15 \%$ of women in reproductive age and may be expressed in peritoneum, ovary or as deeply infiltrating lesions. Symptoms could be pelvic pain, which includes deep dyspareunia, dysmenorrhea and aciclic pain, infertility and intestinal and urinary cyclical changes. The diagnostic suspicion is made through suggestive clinical and image exams, but it depends on the confirmation by surgical procedures such as video-laparoscopy that allows viewing the pelvis and obtaining tissues samples for further histological analysis. Some researches had been done about the delay on diagnosis since the first symptoms and one of the major causes is the lack of knowledge of the patients and even the doctors about the disease, beyond the difficulty of access to laparoscopy procedures at the public health system. In order to find some relation between the sites of the disease and clinical data, many researches have been done and the most evident associations are: deep dyspareunia related to lesions in pouch of Douglas and severe dysmerorrhea related to the presence of pelvic pain in advanced stages of endometriosis. The present paper is a literature review to find some consistent relation between the characteristics of the disease and the symptoms, and to search for the bias of patient's selection or information, that undermined some of the researches. This is an attempt to allow the medical practice to make the diagnosis earlier.
\end{abstract}

KEY WORDS: Infertility. Pelvic pain. Endometriosis.

\title{
REFERÊNCIAS
}

1. Abrão MS, Podgaec S, Izzo CR, Melo PV, Porto RC, Ramos LO, et al. Perfil epidemiológico e clínico da endometriose: estudo de 180 casos. Rev Bras Ginecol Obstet. 1995; 17:779-84.

2. Abrão MS, Podgaec S, Filho BM, Ramos LO, Pinotti $\mathrm{JA}$, de Oliveira RM. The use of biochemical markers in the diagnosis of pelvic endometriosis. Hum Reprod.1997;12:2523-7.

3. Anaf V, Simon P, El Nakadi I, Fayt I, Simonart T, Buxant $F$, et al. Hyperalgesia, nerve infiltration and nerve growth factor expression in deep adenomyotic nodules, peritoneal and ovarian endometriosis. Human Reprod. 2002;17:1895-900.

4. Arruda MS, Petta CA, Abrão MS, Benetti-Pinto CL. Time elapsed from onset of symptoms to diagnosis of endometriosis in a cohort study of Brazilian women. Hum Reprod. 2003;18:756-9.

5. Berkkanoglu M, Arici A. Immunology and endometriosis. Am J Reprod Immunol. 2003;50:48-59.

6. Berkley KJ, Dmitrieva N, Curtis KS, Papka RE. Innervation of ectopic endometrium in a rat model of endometriosis. Proc Natl Acad Sci USA. 2004;101:11094-8.

7. Brosens IA, Brosens JJ. Endometriosis. Eur J Obstet
Gynecol Reprod Biol. 2000;90:159-64.

8. Chopin N, Vieira M, Borghese B, Foulot H, Dousset, $\mathrm{B}$, Coste $\mathrm{J}$, et al. Operative management of deeply infiltrating endometriosis: results on pelvic pain symptoms according to a surgical classification. J Minim Invasive Gynecol. 2005;12:106-12.

9. Chopin N, Ballester M, Borghese B, Fauconnier A, Foulot $\mathrm{H}$, Malartic $\mathrm{C}$, et al. Relation between severity of dismenorrhoea and endometrioma. Acta Obstet Gynecol Scand. 2006;85:1375-80.

10. Dias JA, Podgaec S, Averbach M, Petta CA, Bassi MA, Abrão MS. Time elapsed between the onset of symptoms and the diagnosis of deeply infiltrating endometriosis. In: X Congress Mundial de Endometriose, Melbourne, Austrália, 2008.

11. Fedele L, Parazzini F, Bianchi S, Arcaini L, Candiani GB. Stage and localization of pelvic endometriosis and pain. Fertil Steril. 1990;53:155-8.

12. Fedele L, Bianchi S, Bocciolone L, Di Nola G, Parazzini F. Pain symptoms associated with endometriosis. Obstet Gynecol. 1992;79:767-9.

13. Giudice LC, Kao LC. Endometriosis. Lancet. 2004;364:1789-99. 
14. Harada T, Iwabe T, Terakawa N. Role of cytokines in endometriosis. Fertil Steril. 2001;76:1-10.

15. Meyer R. Ueber den Stand der Frage der Adenomyositis und Adenomyome in Algemeinen und Insbesondere ueber Adenomyositis seroepithelialis und Adenomyometritis sacromatosa. Zentralb Gynakol. 1919;43:745-50.

16. Missmer SA, Cramer DW. The epidemiology of endometriosis. Obstet Gynecol Clin North Am. 2003;30:1-19.

17. Nisolle M, Donnez J. Peritoneal endometriosis, ovarian endometriosis and adenomyotic nodules of the rectovaginal septum are three different entities. Fertil Steril. 1997;68:585-96.

18. Podgaec S, Abrao MS, Dias JA, Rizzo LV, de Oliveira RM, Baracat EC. Endometriosis: an inflammatory disease with a Th2 immune response component. Hum Reprod. 2007;22:1373-79.

19. Sampson JA. Peritoneal endometriosis due to the menstrual dissemination of endometrial tissue into the peritoneal cavity. Am J Obstet Gynecol. 1927; 14:422-69.

20. Szendei G, Hernádi Z, Dévényi N, Csapó Z. Is there any correlation between stages of endometriosis and severity of chronic pelvic pain? Possibilities of treatment. Gynecol Endocrinol. 2005;21:93-100.

21. Ulukus M, Arici A. Immunology of endometriosis. Minerva Ginecol. 2005; 57:237-48.

22. Zondervan KT, Cardon LR, Kennedy SH. What makes a good case-control study? Design issues for complex traits such as endometriosis. Hum Reprod. 2002;17:1415-23. 